\title{
Latin Species Names
}

Acipenser naccarii 202, 209

Acyrthosiphon pisum 177

Adamussium colbecki 130

Aedes aegypti 177

Aegilops tauschii 8

Alburnus alburnus 214, 215

Amphichthys cryptocentrus 191

Anolis carolinensis 72, 79

Anopheles gambiae 177, 183, 215

Apis mellifera 79, 177, 183

Arabidopsis lyrata 14, 84

Arabidopsis thaliana 5, 7, 12, 14, 22, 78, 84,

138, 183

Aspergillus fumigatus 7

Astatotilapia latifasciata 205, 211, 216

Astyanax scabripinnis 211, 214, 215

Bacillus thuringiensis 215

Bathygobius soporator 201

Batrachoides manglae 191

Bombyx mori 63, 71, 79, 177, 183

Bos taurus 187

Brachycome dichromosomatica 215

Caenorhabditis elegans 4, 7, 20, 23, 30, 84, 154, $160,181,183$

Caenorhabditis remanei 84

Candida albicans 11, 12

Candida glabrata 7

Characidium cf. zebra 214

Chionodraco hamatus 214

Cobitis taenia 199

Cryptococcus neoformans 183

Cyphocharax spilotus 214

Danio rerio 79, 183, 199, 205

Daubentonia madagascariensis 147
Diadromus pulchellus 156

Dictyostelium discoideum 15, 183

Donax trunculus 8

Drosophila ananassae 177

Drosophila buzzatii 138

Drosophila erecta 177

Drosophila grimshawi 177

Drosophila melanogaster $4,8,13,23,50,51$, $52,54,56,58,62,63,76,78,81,83,84$, $115,138,159,160,163,165,177,180$, 183

Drosophila mojavensis 177

Drosophila persimilis 177

Drosophila pseudoobscura 177

Drosophila sechellia 177

Drosophila simulans 78,177

Drosophila subobscura 84

Drosophila subsilvestris 8

Drosophila virilis 15, 50, 62, 63, 138, 177

Drosophila willistoni 177

Drosophila yakuba 50, 177

Encephalitozoon cuniculi 13

Entamoeba dispar 78

Entamoeba histolytica 78, 183

Entamoeba invadens 78

Entamoeba moshkovskii 78

Erythrinus erythrinus 201, 205, 207, 214

Eyprepocnemis plorans 8

Fugu rubripes 5

Gasterosteus aculeatus 199

Haemophilus influenzae 114, 119

Halobatrachus didactylus 191

Homo sapiens 138, 183 
Hoplias malabaricus 11, 201, 206, 208, 211, 213

Hydra magnipapillata 20

Hyphessobrycon vinaceus 201

Ictalurus punctatus 199

Imparfinis schubarti 205

Kluyveromyces lactis 113

Leporinus elongatus 211, 212

Lilium longiflorum 187

Metynnis maculatus 216

Moenkhausia sanctaefilomenae 215

Monomorium subopacum 8

Mus musculus 183

Musca domestica 8

Muscari commosum 8

Mycoplasma hyorhinis 118

Nasonia vitripennis 177, 215

Neisseria gonorrhoeae 113, 114

Neisseria meningitidis 118

Neurospora crassa 114,115

Notophthalmus viridescens 156

Notothenia coriiceps 207

Onchorhynchus kisutch 206

Oncorhynchus mykiss 199, 206

Oreochromis karongae 201, 206

Oreochromis niloticus 199, 201, 202, 203, 206

Oryza australiensis 14,78

Oryza sativa $14,139,183$

Oryzias latipes 199

Oryzomys palustris 80

Palorus ratzeburgii 138, 154, 156

Palorus subdepressus 130, 154, 156

Parauchenipterus galeatus 214

Parodon hilarii 213

Pediculus humanus 177

Pholeuon proserpinae 10

Pimelia elevata 138

Plasmodium falciparum 77

Platypus anatinus 73

Poecilia formosa 214

Poecilia reticulata 206

Porichthys plectrodon 191

Prochilodus lineatus 211, 214, 215
Rhamdia hilarii 214

Rineloricaria latirostris 205

Rumex acetosa 8

Saccharomyces cerevisiae $3,4,7,11,12,20$, $113,121,163,183$

Salmo salar 199, 206

Salmo trutta 206

Salvelinus fontinalis 164

Schizosaccharomyces pombe 11, 12, 157, 158, 159,160

Scilla siberica 8

Secale africanum 8

Secale cereale 8, 215

Secale montanum 8

Secale silvestre 8

Silene latifolia 8

Solea senegalensis 190

Sphoeroides spengleri 214

Squalius pyrenaicus 204

Steindachneridion scripta 205

Symphysodon aequifasciatus 207

Symphysodon haraldi 208

Takifugu rubripes 170, 199, 203

Tenebrio molitor 136

Tetraodon nigroviridis 5, 7, 13, 79, 199, 203, 216

Thalassophryne maculosa 191

Tribolium audax 137

Tribolium castaneum 63, 78, 127, 160, 163, 165,177

Tribolium madens 9, 137

Trichomonas vaginalis 77

Triportheus nematurus 211

Trypanosoma brucei 187

Xenopus tropicalis 79

Xiphophorus maculatus 199, 211, 212

Yarrowia lipolytica 183

Zamia paucijuga 134

Zea mays 4, 78 